\title{
Fractura vertical de atlas: reporte de caso y revisión de la literatura
}

Huber S. Padilla-Zambrano1,2,3, Yancarlos Ramos-Villega ${ }^{1,2}$, Daniela López-Cepeda1,2, Willem Guillermo Calderón-Miranda ${ }^{6}$, Andrea Andrade-López ${ }^{1,2}$, Laura Rodríguez-Reyes ${ }^{1,2}$,

Andrés M. Rubiano ${ }^{5}$, A. Pacheco-Hernández ${ }^{4}$, Luis Rafael Moscote-Salazar ${ }^{2,3,4}$

1 Estudiante de Medicina. Facultad de Medicina, Universidad de Cartagena, Cartagena de Indias, Colombia.

2 Centro de Investigaciones Biomédicas (CIB), Facultad de Medicina, Universidad de Cartagena, Cartagena, Colombia.

3 RED LATINO Organización Latinoamericana de Trauma y cuidado Neurointensivo, Bogotá, Colombia.

4 Médico. Especialista en Neurocirugía. Faculta de Medicina, Universidad de Cartagena, Cartagena de Indias, Bolívar.

5 Universidad El Bosque, Bogotá, Colombia.

6 Residente de Radiología, Universidad Nacional Autónoma de México, Ciudad de México. Hospital General Dr. Manuel Gea González.

Rev. Chil. Neurocirugía 44: 40-43, 2018

\section{Resumen}

Las fracturas del atlas son infrecuentes y representan entre el 1 al $2 \%$ de todas las fracturas vertebrales. Anatómicamente el atlas está protegido por el occipucio y las estructuras ligamentosas. Por presentar una forma en anillo, es inusual observar una fractura unilateral. Presentamos el caso de un paciente de 25 años quien presento politraumatismo y a quien se le realizo el diagnostico de fractura vertical de atlas recibiendo manejo conservador. Hacemos una revisión de la literatura de los casos publicados hasta la fecha.

Palabras clave: Fractura de atlas, columna vertebral, neurocirugía.

\begin{abstract}
Atlas fractures are infrequent and account for 1 to $2 \%$ of all fractures vertebral fractures. Anatomically the atlas is protected by occiput and ligamentous structures. Because it presents a ring shape, it is unusual to observe a unilateral fracture. We present the case of a 25-year-old patient who presented polytrauma and who was diagnosed with vertical fracture of atlas receiving conservative management. We do a literature review of the cases published to date.
\end{abstract}

Key words: Atlas fracture, spine, neurosurgery.

\section{Introducción}

Las fracturas vertebrales de altas constituyen una entidad poco frecuente, fue descrita por primera en 1920 por Jefferson. Estas representan aproximadamente entre el $2-13 \%$ de las fracturas de la columna en la región cervical ${ }^{1-5}$. Se relacionan con una compresión axial de alta energía, hiperextensión o una combinación de ambas cargas ${ }^{4}$ y estan asociada a caídas y accidentes de vehículos automotores, que en conjunto representan aproximadamente entre el $80-85 \%$ de todos los casos reportados $^{6}$. La clasificación de la fractura se realiza en función de la localización de la fractura y de la lesión en ligamentos ${ }^{2}$. Dentro de las manifestaciones clínicas más frecuentes se encuentran el dolor de cuello, espasmo muscular y movilidad reducida del cuello ${ }^{3}$. La hinchazón de tejidos blandos prevertebrales en la vista lateral o el desplazamiento lateral son indicadores importantes en el diagnóstico. El uso de tomografía computarizada - TC permite evidenciar la fractura de atlas ${ }^{2}$. Las opciones de tratamientos dependen de las lesiones asociadas, la integridad del ligamento 
transversal y la inestabilidad ${ }^{3}$. Se presente el caso de una fractura vertical de atlas y una revisión de literatura.

\section{Descripción del caso}

Paciente masculino de 25 años sin antecedentes mórbidos de importancia quien es llevado al servicio de urgencia por presentar politraumatismo secundario a accidente en motocicleta a alta velocidad. Los signos vitales al ingreso eran TA 140/80 mmHg, FC 68 Ipm, FR 20 rpm, sin déficit neurológico aparente. Se realiza TAC de cerebro que es reportada dentro de parámetros normales, por lo que el servicio de neurocirugía descarta patología quirúrgica. A las 24 horas, el paciente presenta dolor cervical intenso y se realiza TAC de unión craniocervical con reconstrucción tridimensional que evidencia fractura vertical de atlas. (Figuras 1 y 2). Es revalorado por neurocirugía quien considera manejo conservador con collar cervical rígido durante 1 mes. Se realizó RM de columna cervical que descarto lesiones asociadas. (No disponible). El paciente continuo asintomático y en controles ambulatorio por neurocirugía.

\section{Discusión}

El atlas es la primera vertebral cervical (C1) y una estructura fundamental en la unión craneocervical, que actúa como una estructura de transición entre el occipucio y la columna cervical. En comparación con cualquier nivel de la columna vertebral, la arquitectura de la $\mathrm{C} 1$ permite una diversidad de movimientos y flexibilidad, por estas características una aplicación de fuerza traumática puede generar una fractura única que se debe considerar por separado en relación con otras fracturas en los diferentes niveles de la columna vertebral ${ }^{7}$.

\section{Anatomía de atlas}

El atlas es una estructura de anillo simple con 2 masas laterales puenteadas anterior y posteriormente por arcos neurales. El desarrollo embriológico de C1 resulta en la falta de un cuerpo vertebral propiamente dicho, que se incorpora en $\mathrm{C} 2$ como el proceso denso u odontóide. El odontoide se articula con el arco anterior de C1 para formar una verdadera articulación sinovial. Las

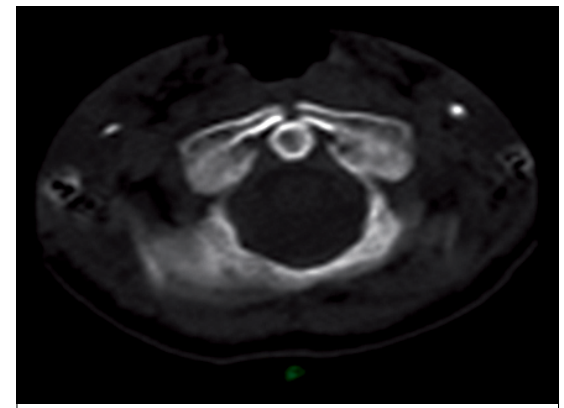

Figura 1. TAC de columna cervical que muestra fractura cervical anterior.

facetas articulares superiores de las masas laterales son cóncavas y articuladas con los cóndilos occipitales convexos. Las facetas inferiores articulan con las facetas articulares superiores de C2 para formar una articulación sinovial bicóncava ${ }^{7}$.

El ligamento de las capsulas de la faceta, el ligamento longitudinal anterior, la membrana tectorial (continuación del ligamento posterior), ligamentos apicales, ligamentos alares y el ligamento cruzado permiten una estabilidad de la unión craneocervical. Además, el complejo mioligamentoso (que consiste en la membrana atlanto-occipital), el ligamento interespinoso, ligamentum nuchae, los músculos rectos posteriores mayores y menores y los músculos superior e inferior obliquus capitis, también contribuir a la estabilidad de la unión. En cada uno de los 2 procesos transversales de $\mathrm{C} 1$ contiene un agujero que acomoda la arteria vertebral a medida que asciende por la columna vertebral. Las arterias vertebrales hacen una curva aguda póstero-medial alrededor de la cápsula articular occipital-C1 que recorre el aspecto superior del arco posterior $\mathrm{C} 1$, que forma una ranura sulcal, antes de que las arterias entren en la duramadre ${ }^{7}$.

\section{Clasificación de la fractura de atlas}

No se ha determinado una clasificación universal para las fracturas de atlas, sin embargo se han observado a lo largo del tiempo distintas propuestas de clasificación. Gehweiler et al., con base a lo propuesto por Jefferson clasificaron la fractura de atlas en 5 tipos I, II, III, IV y V asociadas a afección del arco posterior, anterior, facturas bilaterales del arco posterior asociadas a una fractura unilateral o bilateral del arco anterior, implican la masa y fracturas transversales del arco anterior,

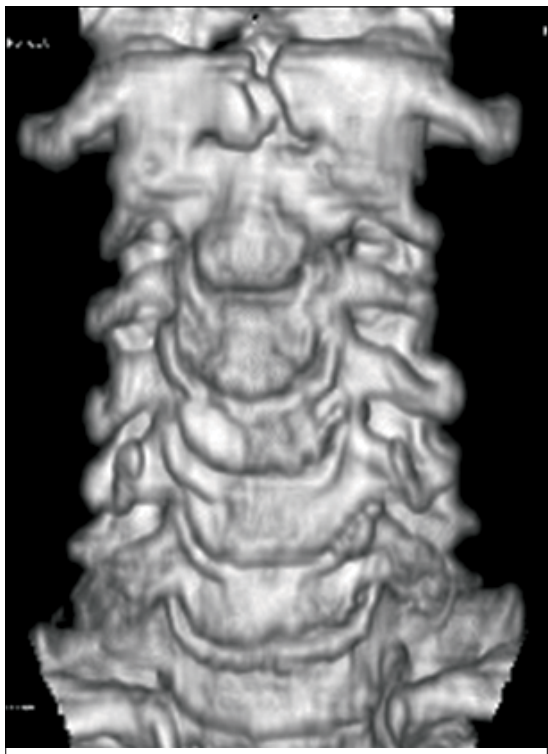

Figura 2. Reconstruccion $3 D$ de columna cervical que muestra fractura cervical anterior.

respectivamente ${ }^{8}$.

Por otra parte, Landells y Van Peteghem en su propuesta de clasificación plantearon 3 tipos: La tipo I involucran solo el arco anterior o posterior, la tipo II involucra fracturas de ambos arcos anterior y posterior y la tipo III es una fractura de la masa lateral con o sin un fractura del $\operatorname{arco}^{9}$. Por último, Levine et al., observaron 3 tipos de fracturas de atlas: bilaterales del arco posterior, masa lateral y de Jefferson o ruptura ${ }^{4}$.

\section{Epidemiología}

Las fracturas cervicales, a diferencia de las fracturas en la primera vertebra son frecuentes, estas representan aproximadamente el $25 \%$ de las lesiones craneocervicales, entre el $3-13 \%$ de las lesiones de la columna cervical y entre el $1-2 \%$ de todas las fracturas en la columna vertebral ${ }^{5,7}$. Es más frecuente en el sexo masculino con un $60 \%$ aproximadamente de los casos reportados y la media de edad se encuentra entre los 30 y 52 años $^{5}$

En el año 2015, Matthiessen y Robinson encontraron en los 1.537 casos de fractura de atlas reportados entre 1997-2011, que el 56,5\% correspondían al sexo masculino y el $43,5 \%$ al sexo femenino, con una edad media de 64 años. Las fracturas de altas representaron el $10,6 \%$ de las fracturas de la región cervical. La incidencia anual casi se duplica en el período del estudio y en el año 2011 fue de 17 por cada 
1.000.000 de habitantes. Además, en la población de edad avanzada se observó un aumento de la incidencia ${ }^{5}$.

\section{Etiología}

Las fracturas del atlas son el resultado de una carga ya sea simétrica, asimétrica o axial en el occipucio y las masas laterales de $\mathrm{C}_{1}^{2}$. Son causadas principalmente por caídas en la parte superior de la cabeza o accidentes de tránsito $^{2,5,6}$ y están relacionadas con cargas externas transmitidas al complejo de la cabeza $^{6}$, asociándose con lesiones del ligamento transversal y fracturas del cuello no contiguas ${ }^{3}$. En muchos estudios de casos clínicos se atribuyó a la fractura de atlas la comprensión axial enérgica y fuerzas que actúan ya sea de forma individual o en conjunto como la hiperflexión y la hiperextensión ${ }^{4,7}$.

\section{Fisiopatología}

Las fuerzas axiales actúan en combinación con flexión, extensión y flexión lateral del occipucio ${ }^{3,4,7}$. La faceta articular superior del atlas está inclinada en una dirección mediocaudal, mientras que las facetas articulares inferiores están orientadas en dirección mediocraneal. Las 2 masas laterales en forma de cuña son sostenidas por los arcos anterior y posterior. Como resultado de esta distribución se ejerce una fuerza radial sobre las masas laterales que buscan expandir el anillo, estas al superar la capacidad elástica ósea produce las fracturas ${ }^{3,7}$. Debido a estas características anatómicas, la mayoría de las lesiones resultan en al menos 2 roturas. Se ha sugerido que la susceptibilidad anatómica de algunos segmentos del anillo (anterior y posterior a las masas laterales), así como la elasticidad de los ligamentos intervertebrales pueden contribuir a la aparición de las fracturas. En adultos, la unión del arco posterior y la masa lateral en $\mathrm{C} 1$, donde cruza la arteria vertebral, se considera más débil ${ }^{2,3,7}$

La densidad mineral ósea se asoció significativamente con las fuerzas axiales máximas al fracaso, lo que apunta a que las fracturas del atlas pueden acontecer como fracturas osteoporóticas $^{6,7}$.

Otro mecanismo es la transmisión de la fuerza desde el vértice, a través de los cóndilos occipitales hasta la columna vertebral. En este la resistencia a la fuerza exhibida por la columna vertebral transita a través de las articula- ciones atlanto-axiales, por lo tanto, el atlas se somete a dos fuerzas que van a través de él en direcciones opuestas y en líneas divergentes. La dirección de esta fuerza es horizontal, y el resultado neto del aplastamiento del atlas es, por lo tanto, una extensión lateral, es decir, una separación de las dos masas laterales entre sí y una fractura de tensión consecuente en uno o más de los puntos débiles en el anillo de atlas ${ }^{10}$.

Por otro lado, la desaceleración abrupta de la cabeza y la transferencia de carga desde el momento del torso hasta la columna cervical superior son capaces de producir la fuerza de cizallamiento anterior y el desplazamiento hacia adelante de las guaridas y el fragmento óseo del arco anterior del atlas en relación con el anillo $\mathrm{C} 1$ ocasionando la fractura ${ }^{4}$.

\section{Manifestaciones clínicas}

Los pacientes que sufren este tipo de fractura presentan síntomas como espamos en los músculos cervicales, dolor de cuello y disminución del rango de movimiento ${ }^{2,3,7,10}$. Además, pueden presentar hinchazón retrofaríngea que puede causar disfagia. La neuralgia y parestesia se pueden presentar y están relacionadas con la compresión o lesión del nervio C2. También se han reportado casos donde hay perdida transitoria de la conciencia, visión doble o signos y síntomas de isquemia de la fosa posterior, debido a lesiones disecantes de la arteria vertebral ${ }^{7,10}$.

\section{Diagnostico}

La historia clínica y el examen físico deben contener detalles sobre el mecanismo de lesión o trauma y una evaluación neurológica completa ${ }^{1}$. La evaluación inicial debe hacerse con radiografía simple, aunque esta no siempre es útil para la detección de fracturas con múltiples fragmentos ${ }^{2}$. A menudo los indicadores notables son la hinchazón de los tejidos blandos prevertebrales en la vista lateral o el desplazamiento lateral en la vista de boca abierta ${ }^{1,2}$. La vista odontóide de boca abierta muestra la superposición de las facetas $\mathrm{C} 1$ y $\mathrm{C} 2$, el desplazamiento de la masa lateral de C1 sobre las facetas de C2. En la vista lateral, se observa un intervalo atlantodental (ADI, por sus siglas en inglés) mayor de $3 \mathrm{~mm}$ en adultos y mayor de $5 \mathrm{~mm}$ en niños. Las fracturas del arco anterior a menudo son difíciles de diagnosticar a partir de las radiografías simples, sin embargo las proyecciones oblicuas pueden mejorar las vistas de ambos arcos del anillo atlas ${ }^{1,7}$.

El diagnostico se hace principalmente con $\mathrm{TC}^{1,2}$. Las imágenes sagitales reconstruidas pueden mostrar fracturas ocultas horizontales del arco anterior. Los cortes axiales pueden mostrar lesiones del ligamento transversal incluso cuando la extensión de masa lateral es inferior a $7 \mathrm{~mm}^{7}$.

La RM de alta resolución es la modalidad de elección para evaluar la integridad del ligamento transversal ${ }^{7,11}$. Dickman et al., describieron dos tipos de lesiones transversas aisladas del ligamento atlantal en la RM. En el Tipo I, el ligamento se lesiona en ausencia de la fractura del atlas. El tipo II implica una fractura de avulsión del atlas en la inserción del ligamento atlantal transverso $^{12}$.

Tanto en la radiografía y la TC, las hendiduras posteriores del arco pueden causar problemas de diagnóstico ocasionales, estas pueden diferenciarse de las fracturas agudas por la estabilidad de la imagen en el tiempo y la presencia de márgenes corticales. Por otro lado, las fracturas agudas suelen tener márgenes afilados y no corticales. La RM puede ser útil para diferenciar entre los pacientes con fracturas agudas y los de las variantes normales ${ }^{2,13}$.

\section{Tratamiento}

El tratamiento de las fracturas de atlas está basado principalmente en la forma de presentación de la fractura, es decir, si esta ocurre de forma aislada o en conjunto con otra fractura de la columna cervical ${ }^{2,7}$, siendo la combinación más frecuente la fractura de $\mathrm{C} 1-\mathrm{C}^{7}$. En el caso de las fracturas de atlas aisladas el tratamiento de elección es la inmobilización externa de la unión craneocervical durante 8 a 12 semanas con un cuello cervical firme, esto es siempre y cuando se haya descartado la inestabilidad ${ }^{2,7}$. En caso de inestabilidad después de la de inmovilización externa se debe realizar una fusión occipito-C2 o una fusión $\mathrm{C} 1-\mathrm{C} 2$ para evitar el daño neurológico ${ }^{7,10}$

Cuando hay lesiones neurológicas se debe principalmente a fracturas del atlas en conjunto con otra fractura de la columna cervical y frecuentemente se relacionan a ruptura del ligamento transverso. Como se mencionó anteriormente las fracturas que se presentan en la mayoría de casos son las 
combinadas de $\mathrm{C} 1-\mathrm{C} 2$, para este tipo de fracturas no hay normas o directrices establecidas para su tratamiento, pero este se basa principalmente en la naturaleza de la fractura de C2 y en la integridad del ligamento transverso. La inmovilización externa es recomendada en casos de estabilidad al igual que en las fracturas aisladas; si se presentan fracturas de odontoides tipo II asociadas con una inestabilidad mayor a $5 \mathrm{~mm}$ o fractura de ahorcado asociada con una angulación mayor al $11 \%$ de C2-C3 es recomendada la fijación quirúrgica y la fusión ${ }^{3,7}$.

\section{Conclusiones}

Las fracturas del atlas son infrecuentes y pueden pasar desapercibidas en le evaluación inicial de los pacientes.
Debemos tener un alto nivel de sospecha para descartar siempre este tipo de fracturas. El manejo conservador fue efectivo en nuestro caso tal como ha sido descrito en la literatura.

Recibido: 10 de septiembre de 2017 Aceptado: 28 de octubre de 2017

\section{Referencias}

1. Srinivas S, Harshavardhana N, Bayley E, Mehdian SMH. Isolated vertical fracture of the anterior arch of the atlas-Case report. Inj Extra. 2009;40(8):152-4.

2. Inaoka T, Ohashi K, El-Khoury GY. A single fracture in the atlas ring: Report of two cases and a review of the literature. Emerg Radiol. 2007;14(3):191-4.

3. Ivancic PC. Atlas Injury Mechanisms During Head-First Impact. Spine (Phila Pa 1976). 2012;37(12):1022-9.

4. Ivancic PC. Plough fracture of the anterior arch of the atlas: A biomechanical investigation. Eur Spine J. 2014;23(11):2314-20.

5. Matthiessen C, Robinson Y. Epidemiology of atlas fractures - A national registry-based cohort study of 1,537 cases. Spine J. 2015;15(11):2332-7.

6. Gebauer M, Goetzen N, Barvencik F, Beil FT, Rupprecht M, Rueger JM, et al. Biomechanical analysis of atlas fractures: a study on 40 human atlas specimens. Spine (Phila Pa 1976). 2008;33(7):766-70.

7. Kakarla UK, Chang SW, Theodore N, Sonntag VKH. Atlas fractures. Neurosurgery. 2010;66(SUPPL. 3):6-8.

8. Gehweiler J, Duff D, Salutario M, Miller M, Clark M. Fractures of the atlas vertebra. Skelet Radiol. 1976;23:97-102.

9. Landells CD, Van Peteghem PK. Fractures of the atlas: classification, treatment and morbidity. Spine (Phila Pa 1976). 1988 May;13(5):4502.

10. Jefferson G. Fracture of the atlas vertebra. Report report of four cases, and a review of those previously recorded. Br J Surg. 1919;7:40722.

11. [No authors listed]. Isolated Fracture of the Atlas In Adults. Neurosurgery. 2002;50(3 Suppl):120-4.

12. Dickman CA, Greene KA, Sonntag VK. Injuries involving the transverse atlantal ligament: classification and treatment guidelines based upon experience with 39 injuries. Neurosurgery. 1996 Jan;38(1):44-50.

13. Vaughan T, West C. Isolated Vertical Fracture through the Anterior Atlas Arch: A Previously Unreported Fracture. Emerg Radiol. 1998;5(4):259-62.

\section{Correspondencia:}

Dr. Luis Rafael Moscote-Salazar

Universidad de Cartagena, Cartagena de Indias, Colombia.

mineurocirujano@aol.com 